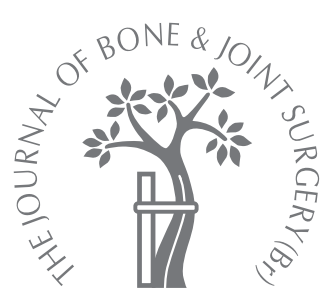

T.-J. Cho,

I. H. Choi,

S. M. Lee,

C. Y. Chung,

W. J. Yoo,

D. Y. Lee,

J. W. Lee

From Seoul National University Children's Hospital, Seoul, Korea

n- T.-J. Cho, MD, Associat Professor

I. H. Choi, MD, Professor

S. M. Lee, MD, Orthopaedic

Resident

W. J. Yoo, MD, Assistant

Professor

D. Y. Lee, MD, Clinical

Fellow

Department of Orthopaedic

Surgery

Seoul National University

Children's Hospital, 28

Yeongeon-dong Jongno-gu,

Seoul 110-744, Korea.

II C. Y. Chung, MD, Professo Department of Orthopaedic

Surgery

Seoul National University Bundang Hospital, 300 GumiDong, Bundang-gu,

Seongnam-si, Gyeonggi-do 463-707, Korea.

In. J. Lee, PhD, Professor Department of Statistics Korea University, 5-1 Anamdong, Sungbuk-gu, Seoul 136701, Korea

Correspondence should be sent to Professorr I. H. Choi; e-mail: inhoc@snu.ac.kr

(C)2008 British Editorial Society of Bone and Joint Surgery doi:10.1302/0301-620X.90B4 $20153 \$ 2.00$

$J$ Bone Joint Surg $[\mathrm{Br}]$ 2008;90-B:488-93. Received 28 August 2007; Accepted after revision 21 December 2007

\title{
Refracture after Ilizarov osteosynthesis in atrophic-type congenital pseudarthrosis of the tibia
}

\begin{abstract}
We investigated patterns of refracture and their risk factors in patients with congenital pseudarthrosis of the tibia after Ilizarov osteosynthesis. We studied 43 cases in 23 patients. Temporal and spatial patterns of refracture and refracture-free survival were analysed in each case. The refracture-free rate of cumulative survival was $47 \%$ at five years and did not change thereafter. Refracture occurred at the previous pseudarthrosis in 16 of 19 cases of refracture. The risk of refracture was significantly higher when osteosynthesis was performed below the age of four years, when the tibial cross-sectional area was narrow, and when associated with persistent fibular pseudarthrosis. Refracture occurs frequently after successful osteosynthesis in these patients. Delaying osteosynthesis, maximising the tibial cross-sectional area and stabilising the fibula may reduce the risk of refracture.
\end{abstract}

Although the primary state of union in the treatment of congenital pseudarthrosis of the tibia has improved greatly with the use of intramedullary $\operatorname{rods}^{1}$ or the Ilizarov methods, ${ }^{2,3}$ challenging problems frequently ensue and jeopardise function. These include leglength discrepancy, multilevel and multidirectional tibial deformity, foot deformity, associated fibular pseudarthrosis, and subsequent ankle valgus. ${ }^{3,4}$ Refracture is the most serious complication that interferes with function, and may result in re-establishment of the pseudarthrosis. Moreover, current treatments do not completely prevent refracture. ${ }^{1-11}$ There has been no systematic analysis of refracture in patients with congenital pseudarthrosis of the tibia. In the majority of reports, refracture frequencies are described as the rate of refracture over a certain period of follow-up. However, as the risk of refracture persists until or after skeletal maturity, survival analysis offers a more appropriate means of describing this complication, as it provides a temporal profile of refracture and enables the analysis of the risk factors. With regard to risk factors for refracture, cross-sectional area of the healed segment, ${ }^{2}$ residual angular deformity ${ }^{2}$ and removal of an intramedullary $\operatorname{rod}^{12}$ are suggested, but have not been supported by statistical data.

The authors have used the Ilizarov method for the treatment of congenital pseudarthrosis of the tibia since 1989 and achieved high rates of union. ${ }^{3}$ However, subsequent complications such as refracture and the development of angular deformity, remain unavoidable. Their prevention and treatment are important, especially as high rates of bony union of congenital pseudarthrosis of the tibia have been achieved. We therefore investigated the temporal and spatial patterns of refracture, and the associated risk factors in a group of relatively homogeneous patients with respect to disease type and treatment protocol.

\section{Patients and Methods}

This retrospective study was approved by the Institutional Review Board of Seoul National University Hospital. A total of 23 consecutive patients treated since 1989, who had presented with atrophic congential pseudarthrosis of the tibia (Boyd type $\mathrm{II}^{13}$ or Crawford type II- $\mathrm{C}^{14}$ ) were studied. There were 13 girls and ten boys. The right side was involved in 12 and the left in 11. A total of 19 had neurofibromatosis type I. The patients were followed for a mean of 9.2 years $(2.3$ to 16.7$)$. Of the 23 patients, 12 presented at a mean age of 3.2 years $(0.8$ to 6.8$)$ having had no previous surgical treatment. The remaining 11 (mean age 6.4 years, 2.0 to 11.8 ) had been treated surgically at other hospitals before referral, by the Ilizarov method in five patients, onlay bone graft in two and intramedullary rodding in two. The treatment history of two patients was incomplete.

All operations were performed or supervised by a single surgeon (IHC). The procedure for established pseudarthrosis consisted of meticulous and complete resection of the sclerotic 


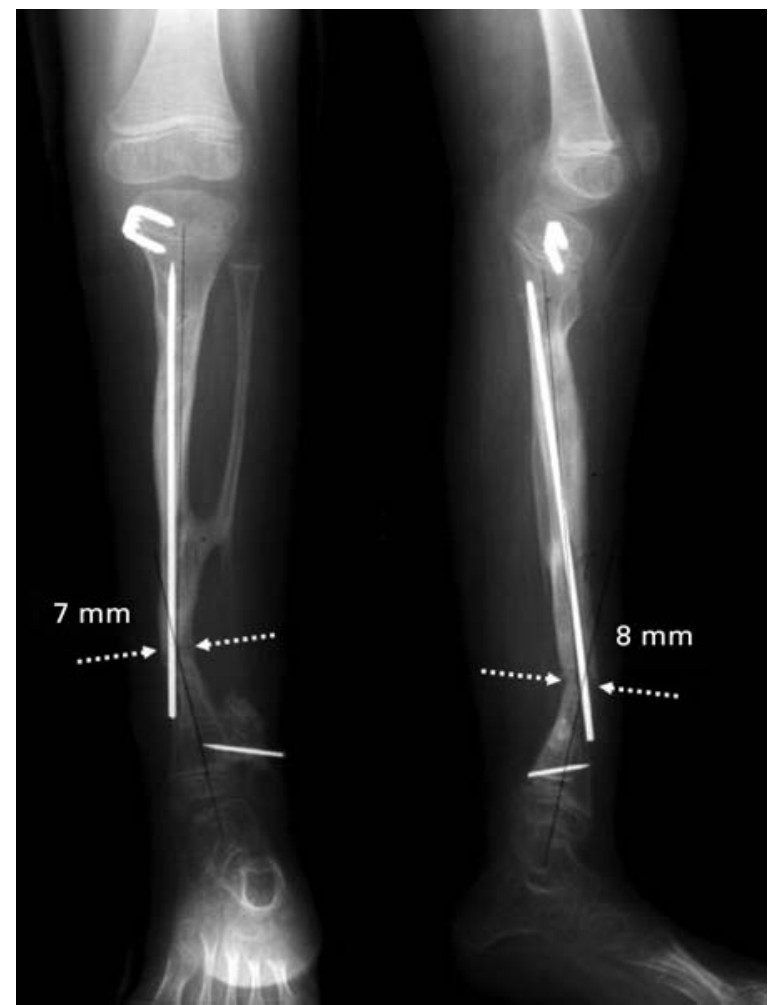

Fig. 1

Radiographs of a 5.5-year-old boy with neurofibromatosis type 1associated congenital pseudarthrosis of the tibia who sustained a third fracture at the previous pseudarthrosis site. The tibial diaphyseal cross-sectional area was assumed to be $56 \mathrm{~mm}^{2}$, the product of the narrowest diameters on anteroposterior and lateral projections.

bone edges and surrounding fibrous hamartoma, and reopening of the medullary canals. An Ilizarov fixator was applied using only tensioned smooth wires. Acute compression at the pseudarthrosis was used in 19 patients and internal bone transport in the remaining four. Generous autogenous bone grafting was an essential part of the operation. Cancellous chips were placed around pseudarthroses and surrounded by strips of cortical onlay graft, which were tied with wires or non-absorbable sutures. Bone graft was harvested from the iliac crest in 27 cases of osteosynthesis, but in six cases the normal contralateral tibial cortex was also used. Bone marrow aspirate alone was injected in two cases of osteosynthesis and demineralised bone matrix in one.

Bony union was defined as restoration of cortical integrity, confirmed by plain radiography and full weightbearing without a walking aid. A total-contact short leg brace was recommended until skeletal maturity. However, patient and parent compliance varied.

Refracture was defined as a deterioration of weightbearing ability with progressive angulation, or an obvious fracture on a plain radiograph. There were 19 refractures in
12 patients treated using an Ilizarov fixator. Repeated resection of fibrous hamartoma and autogenous bone grafting were performed in 15 refractures. In the remaining four, in whom the refracture site was not dysplastic and only minimally displaced, closed compression osteosynthesis using the Ilizarov fixator was performed.

Each attempted osteosynthesis for pseudarthrosis at the first presentation in 23 patients, refracture in 19 or nonunion of tibial lengthening in one was considered as a separate case, a total of 43 of which were divided into two groups: those who sustained refracture during follow-up comprised the refracture group and the remainder formed the no-fracture group. Temporal and spatial patterns of refracture were analysed. The factors examined regarding refracture-free survival were age at the time of operation, cross-sectional area of the tibial diaphysis, status of the fibula, the presence of an intramedullary rod and association with neurofibromatosis type 1 .

Refracture-free survival according to age was analysed in two ways. First, the two groups were compared with respect to age at operation. Also, those undergoing osteosynthesis over (28 cases) and under (15 cases) four years of age were compared. This age was selected because it gave the most significant difference.

The narrowest diameters of the tibial shafts were measured on anteroposterior (AP) and lateral radiographs taken at the time of refracture in this group (Fig. 1) and during the second post-operative year in the no-fracture group. The second year was selected because the median time to refracture was 1.8 years post-operatively. The products of the diameters on AP and lateral projections were regarded as representing cross-sectional areas and were compared between the two groups. Also, those with a cross-sectional area larger or smaller than the median value of $182 \mathrm{~mm}^{2}$ were compared with respect to refracture-free survival.

Associated fibular pseudarthrosis was seen in 34 cases and was treated in different ways. In nine, it was neglected, concentrating on the tibial osteosynthesis alone. Osteosynthesis of diaphyseal fibular pseudarthrosis was performed in three cases, and tibiofibular synostosis, either distal only or proximal/distal together in 22 , one of which failed. The whole 43 cases were dichotomised according to the fibular status at the completion of tibial bony union as either persistent fibular pseudarthrosis due to neglect or failed osteosynthesis (ten cases), or intact fibular or distal fibular stabilisation by tibiofibular synostosis (33). Refracture-free survival was compared between these two groups.

An intramedullary Steinmann pin was inserted at the time of osteosynthesis in 29 cases in order to minimise shear stress. It was removed in ten cases after tibial bony union and in the remaining 19 it was repositioned to mobilise the ankle joint and was retained in the medullary canal when the Ilizarov frame was removed. The criteria for placement and retention of the rods were not prospectively defined nor randomised. Refracture-free survival was compared between those with and without an intramedullary rod. 


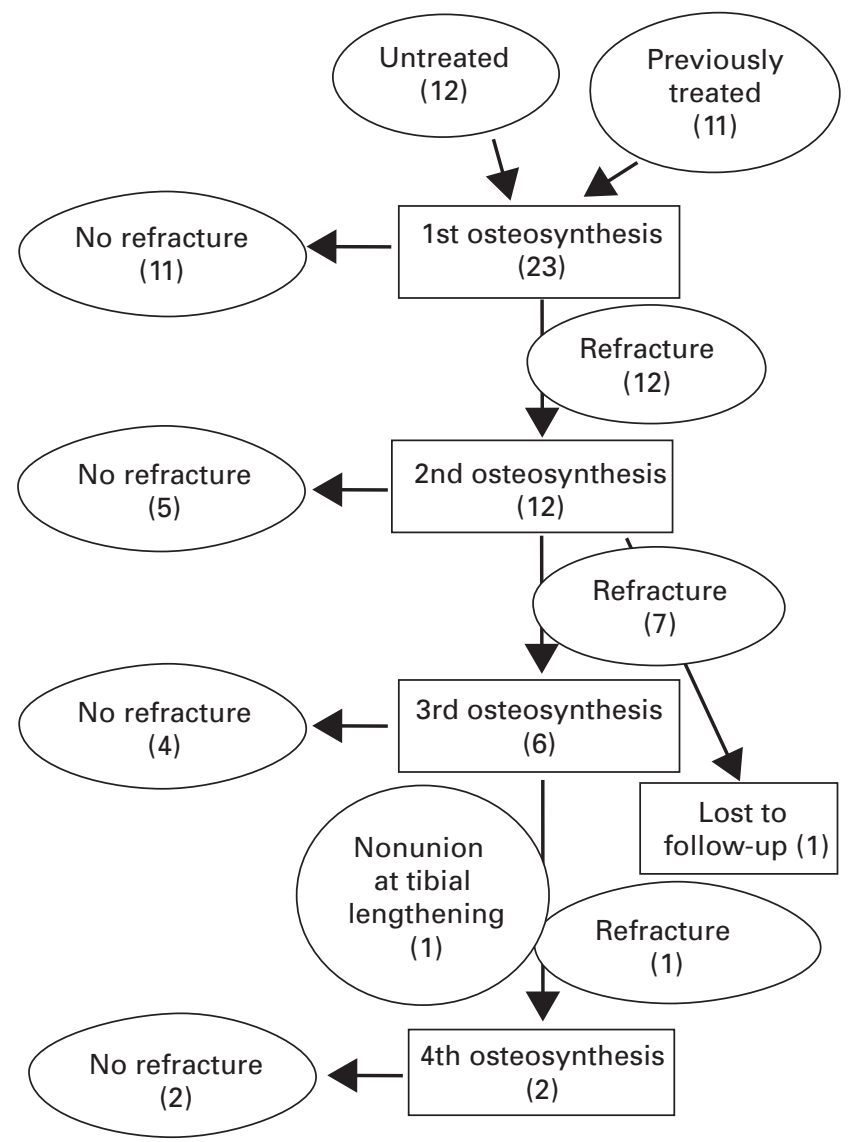

Fig. 2

Flow chart of the cases in this study. A total of 43 cases of osteosynthesis were included.

Survival analysis was performed using the Kaplan-Meier method and the log-rank test was used to compare refracture-free survival rates between the two groups. Comparisons of the refracture and no-fracture groups with respect to age and cross-sectional area were performed using the two-sample $t$-test. Cox's regression using the proportional hazards model was used to determine the effects of risk factors on refracture-free survival. All statistical analyses were performed using SPSS version 12.0 (SPSS Inc., Chicago, Illinois) and a p-value $<0.05$ was considered statistically significant.

\section{Results}

Osteosynthesis was successfully achieved by Ilizarov treatment in all 23 patients with an established pseudarthrosis. However, 12 had a refracture during follow-up. A second Ilizarov treatment again achieved successful union in all these cases, but seven had a second refracture. A third Ilizarov treatment achieved union in six of these. The remaining patient was lost to follow-up because of emigration. No further patients were lost to follow-up. One of the six cases sustained a third fracture and another underwent

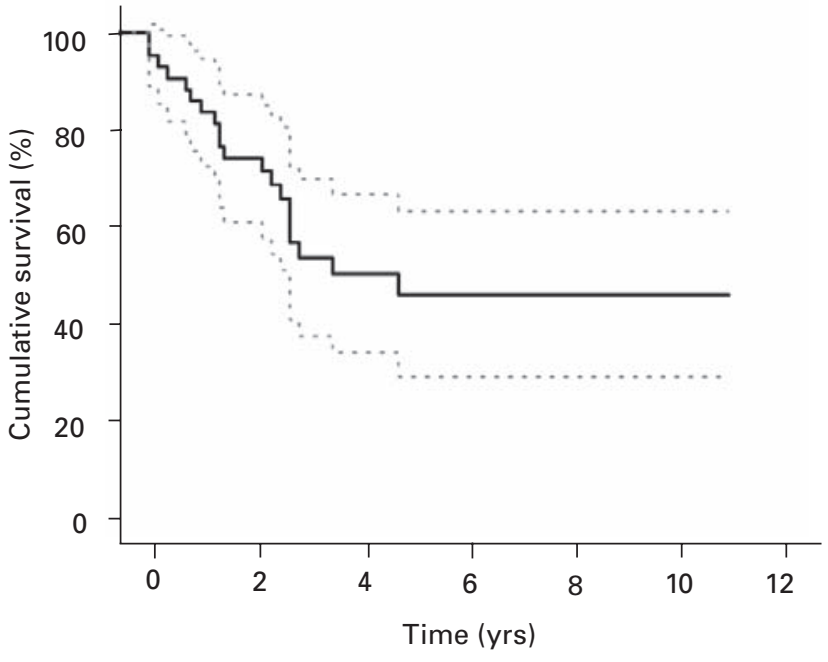

Fig. 3

Kaplan-Meier analysis, with 95\% confidence intervals (dotted line), of refracture-free survival of the 43 cases after osteosynthesis.

proximal tibial lengthening, resulting in nonunion at the distraction gap. These two patients underwent a fourth Ilizarov treatment and bony union was achieved and maintained until the latest follow-up at 2.3 years and nine years respectively (Fig. 2).

The Kaplan-Meier method showed that the refracturefree cumulative survival rate dropped consistently during the first three years to $57 \%$ (95\% confidence interval (CI) 41 to 73 ), and finally reached $47 \%$ (95\% CI 30 to 63 ) at five years (Fig. 3). In the refracture group the mean fracture-free period was 2.1 years $(0.5$ to 4.9$)$. A total of 11 $(58 \%)$ of the 19 refractures occurred within two years, and $16(84 \%)$ within three. In 16 cases $(84 \%)$ refracture occurred at the previous pseudarthrosis. One of the remaining three cases refractured at the Ilizarov pin site and the other two at non-specific locations. In six cases, the previous pseudarthrosis underwent progressive narrowing with periosteal cortical scalloping, and refractures followed insidiously, suggesting a recurrence of fibrous hamartoma.

Of the various factors comparing the refracture and nofracture groups, age at operation, tibial cross-sectional area and fibular status were significantly different (Table I). The mean age at operation in the 43 cases was 5.5 years $(0.8$ to 11.8). It was significantly higher in the no-fracture group. Cumulative survival was also significantly better in those who underwent osteosynthesis over four years of age (Fig. 4).

The cross-sectional area of the tibial diaphysis, measured in 39 cases, was significantly larger in the no-fracture group. Cases were considered according to the median value of $182 \mathrm{~mm}^{2}$ and cumulative survival was significantly better in those with a cross-sectional area larger than this (Fig. 5). 
Table I. Comparison of refracture-free survival

\begin{tabular}{|c|c|c|c|c|}
\hline & \multirow[b]{2}{*}{ Number of cases } & \multicolumn{2}{|c|}{ Survival time (yrs) } & \multirow[b]{2}{*}{ p-value } \\
\hline & & Mean & $95 \%$ confidence interval & \\
\hline Total & 43 & 6.24 & 4.84 to 7.64 & \\
\hline \multicolumn{5}{|l|}{ Age } \\
\hline$\geq 4$ years & 28 & 7.58 & 5.98 to 9.17 & 0.008 \\
\hline$<4$ years & 15 & 4.01 & 2.22 to 5.81 & \\
\hline \multicolumn{5}{|l|}{ Cross-sectional area $^{\dagger}$} \\
\hline$\geq 182 \mathrm{~mm}^{2}$ & 21 & 6.80 & 5.14 to 8.46 & 0.015 \\
\hline$<182 \mathrm{~mm}^{2}$ & 18 & 3.76 & 2.31 to 5.22 & \\
\hline \multicolumn{5}{|l|}{ Fibular status } \\
\hline Intact or stabilised & 33 & 7.00 & 5.46 to 8.55 & 0.008 \\
\hline Pseudarthrotic & 10 & 3.45 & 0.84 to 6.07 & \\
\hline \multicolumn{5}{|l|}{ Intramedullary rod ${ }^{\ddagger}$} \\
\hline Present & 19 & 4.79 & 3.09 to 6.49 & 0.217 \\
\hline Absent & 23 & 7.08 & 5.22 to 8.93 & \\
\hline \multicolumn{5}{|l|}{ Associated with $\mathrm{NF}^{\S}$} \\
\hline Yes & 37 & 5.99 & 4.46 to 7.51 & 0.435 \\
\hline No & 6 & 5.79 & 3.52 to 8.06 & \\
\hline
\end{tabular}

The fibular status was found to affect refracture-free survival significantly. Those with an intact fibula or one stabilised by diaphyseal osteosynthesis or tibiofibular synotosis showed significantly better refracture-free survival than those with a pseudarthrotic fibula due to neglect or failed synostosis (Fig. 6).

Against expectations, cases retaining an intramedullary rod showed shorter refracture-free survival times than those without, although this was not statistically significant (Kaplan-Meier log-rank test, $\mathrm{p}=0.2357$ ). Of 20 refracture cases, 11 had an intramedullary rod at refracture and among the 19 cases retaining such a rod, 11 refractured, all at the previous pseudarthroses.

In total, the 19 patients with neurofibromatosis type 1 accounted for 37 of the attempted osteosyntheses with Ilizarov fixators. There was no significant differences in refracture-free survival between those with and without neurofibromatosis type 1 (log-rank test, $\mathrm{p}=0.4269$ ).

Cox's proportional hazards regression analysis indicated that of the three factors found to be statistically significant by log-rank analysis, tibial cross-sectional area and fibular status, showed statistically significant effects $(p=0.001$ and $\mathrm{p}=0.022$ respectively). Their hazard ratios were 0.986 (95\% CI 0.977 to 0.994 ) and 3.997 (95\% CI 1.218 to 13.119), respectively. The age at surgery correlated highly with tibial cross-sectional area $(\mathrm{r}=0.752, \mathrm{p}<0.001)$, and was not found to be significant by Cox's proportional hazards analysis $(\mathrm{p}=0.532)$.

\section{Discussion}

Although high success rates are reported for treatment of pseudarthrosis using current methods, 2,3,6,7,11,12,15-20 refracture still occurs. This study showed that in atrophic congenital pseudarthrosis of the tibia, age at surgery, tibial cross-sectional area and fibular stability were significant factors determining risk of re-fracture. This may provide a basis for understanding the mechanisms of refracture and devising preventative procedures.

Congentital pseudarthrosis of the tibia is not a homogeneous entity but rather is composed of several disease types, according to radiological appearance and temporal progress, ${ }^{13,14}$ which are associated with different prognoses. 6,7,13,21,22 Therefore, comparing the refracture-free survival between groups of mixed types might not be appropriate. However, all cases in this study were classified as atrophic, Boyd type $\mathrm{II}^{13}$ or Crawford type II-C $\mathrm{C}^{14}$ at initial presentation, and are known to be associated with the worst rates of union and greatest functional deficit. 6,7,13,21,22 Moreover, because a single surgeon treated or at least supervised the treatment in all cases, the series represents a relatively homogeneous group.

It is controversial whether early osteosynthesis under three to four years of age is beneficial. Boyd and Sage ${ }^{22}$ recommended operation as soon as the procedure can be undertaken safely, and Joseph et $\mathrm{al}^{10}$ reported better consolidation, less limb-length discrepancy and less pronounced growth abnormalities in patients treated with intramedullary rodding before three years of age. However, others ${ }^{4,23}$ recommend waiting until the child is five to six years old, because they found that the success rate was higher in older children. More specifically, Grill et $\mathrm{al}^{23}$ reported a healing rate of $64 \%$ in children under three and $92 \%$ in those between six and nine years old. The Ilizarov method in very young children is technically more demanding and has been associated with lower rates of success than in older children. ${ }^{7,22}$ In this study, we found no difference in the rate of 


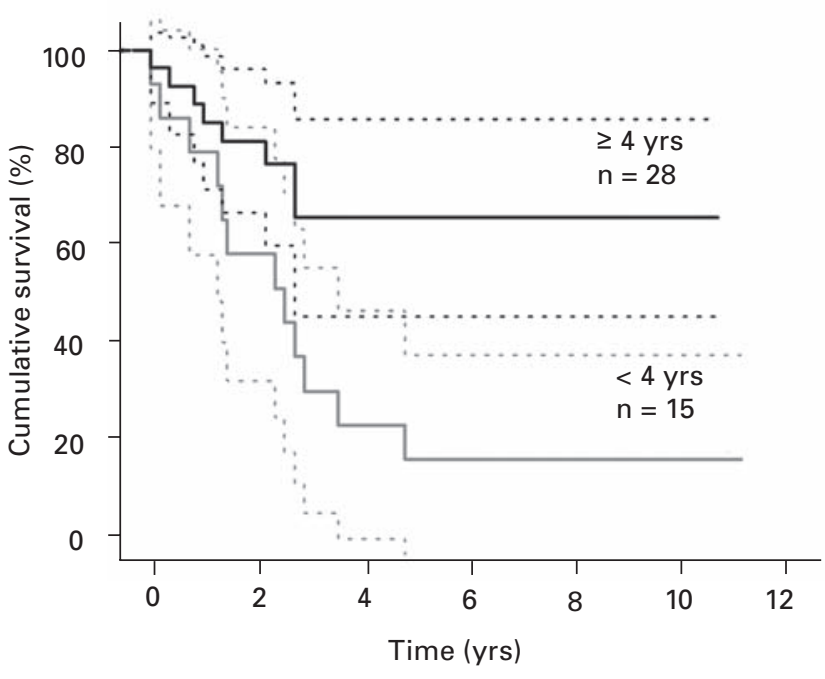

Fig. 4

Kaplan-Meier analysis, with 95\% confidence intervals (dotted line), of refracture-free survival of patients who had osteosynthesis aged $\geq 4$ years versus those aged $<4$ years.

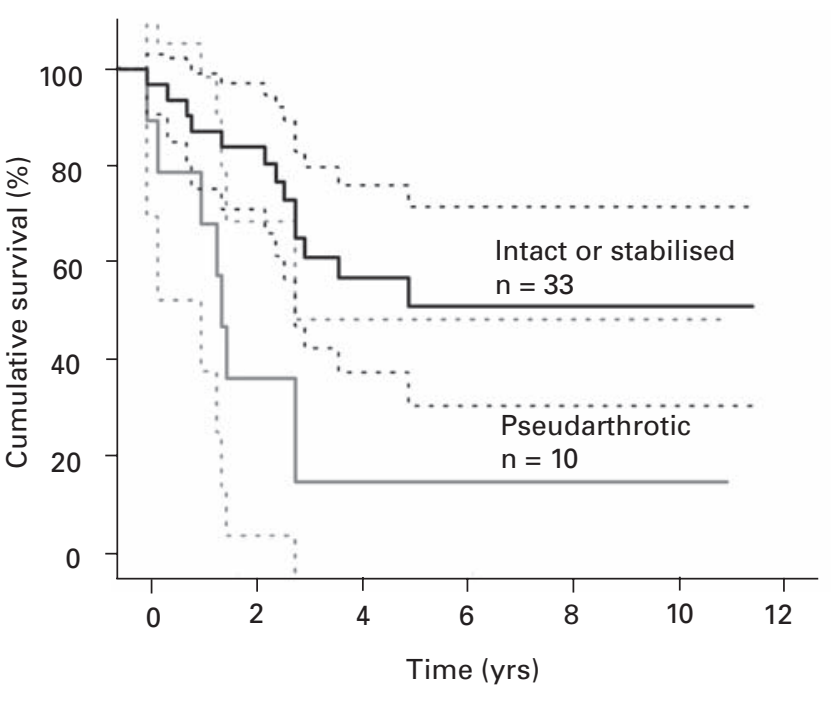

Fig. 6

Kaplan-Meier analysis, with $95 \%$ confidence intervals (dotted line), of refracture-free survival of those with an intact or stabilised fibula versus those with a persistently pseudarthrotic fibula.

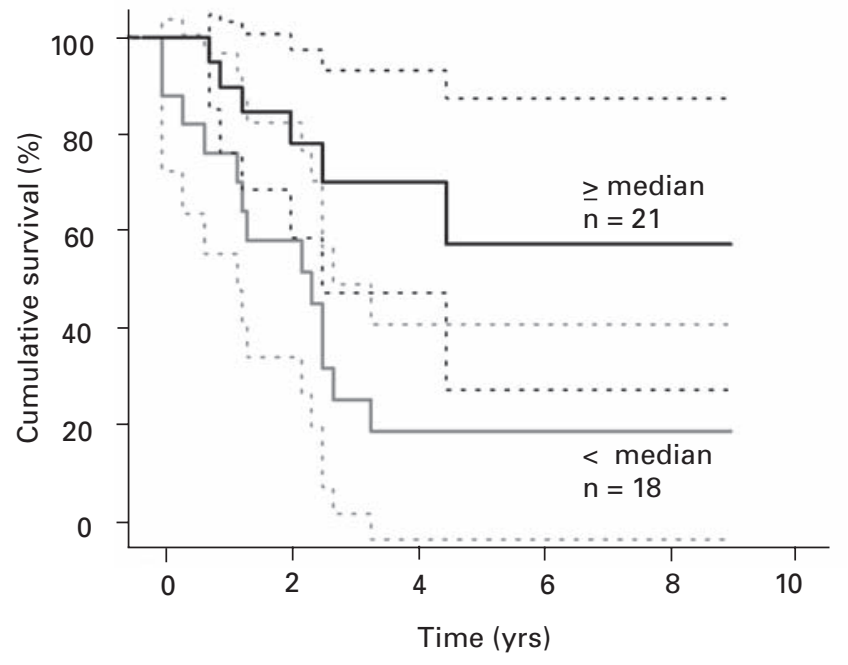

Fig. 5

Kaplan-Meier analysis, with 95\% confidence intervals (dotted line), of refracture-free survival of those patients with a tibial cross-sectional area over the median value of $182 \mathrm{~mm}^{2}$ compared with those with a smaller cross-sectional area.

union according to age. However, a significant difference in refracture rate was observed between those above and those below four years of age. We now postpone osteosynthesis until this age to reduce the risk of subsequent refracture. The effect of age on refracture risk seems secondary to narrow cross-sectional area of the tibia, and was excluded from the Cox's proportional hazards analysis.

Maximisation of the healing cross-sectional area is considered one of the most important principles of treatment ${ }^{2}$ and our findings confirmed this. It is probable that a tibia with a wide cross-sectional area can resist more mechanical stress. Moreover, a narrow or narrowing tibial segment might represent residual or recurrent fibrous hamartoma surrounding a bony union mass. ${ }^{23,24}$ We observed fibrous hamartomatous tissue in all the refracture cases that were explored. We therefore believe that refracture is not just a mechanical event but also a result of a biological process. However, it is not known how to prevent the recurrence of fibrous hamartoma.

The importance of fibular stabilisation in the treatment of congenital pseudarthrosis of the tibia is emphasised by several authors. Tudisco et $\mathrm{al}^{21}$ stated that a fibular pseudarthrosis appeared responsible for most of the poorest results. Persistent fibular pseudarthrosis was also found to be related to failure of tibial union ${ }^{9,11,16}$ and progressive valgus of the ankle. ${ }^{7}$ Moreover, Dobbs et $\mathrm{al}^{12}$ concluded that the prevalence of tibial refracture is higher in patients in whom a fibular pseudarthrosis was not resected. Our study, based on a survival analysis approach, showed that fibular stabilisation by either tibiofibular synostosis or fibular osteosynthesis resulted in significantly better survival. We agree with Coleman and Coleman ${ }^{16}$ that, if necessary, tibiofibular synostosis should be performed, as its benefits outweigh its adverse effects.

Intramedullary rodding is expected to contribute to the prevention of refracture. Dobbs et $\mathrm{al}^{12}$ reported that three of four refractures occurred after rod removal in an attempt to regain ankle movement, and thus discouraged removal of the rod after union. However, this may suggest that it is ankle joint transfixation that prevents refracture, rather than the presence of a rod. In this study, we used intramed- 
ullary rodding primarily to stabilise the pseudarthrosis during healing, and mobilised the ankle when tibial union was achieved. This may be why refracture-free survival was not significantly different between those with or without an intramedullary rod. Moreover, owing to the retrospective nature of this study, persistence of an intramedullary rod was not randomised. Nevertheless, we believe that retention of a rod across the previous pseudarthrosis should have biomechanical benefits, although it cannot prevent the biological process underlying refracture.

Residual angulation at pseudarthroses has been suggested as a primary cause of refracture. ${ }^{2}$ However, we could not analyse this effect as most of our cases healed with a straight diaphysis at the previous pseudarthrosis. Bracing may affect the refracture rate and we recommend a total contact short leg brace in all cases. However, as compliance varied and could not be measured, we excluded that parameter from the analysis. Another limitation of this study is that each episode of osteosynthesis in one patient, although considered as independent cases, cannot be completely independent, thereby leading to potential bias. However, by accepting this compromise, we obtained statistically significant results from a single institute treating this rare condition.

No benefits in any form have been received or will be received from a commercial party related directly or indirectly to the subject of this article.

\section{References}

1. Anderson DJ, Schoenecher PL, Sheridan JJ, Rich MM. Use of an intramedullary rod for the treatment of congenital pseudarthrosis of the tibia. J Bone Joint Surg [Am] 1992;74-A:161-8.

2. Paley D, Catagni M, Argnani F, et al. Treatment of congenital pseudarthrosis of the tibia using the llizarov technique. Clin Orthop 1992;280:81-93.

3. Lee KS, Lee SM, Cho T-J, et al. Outcome results of Ilizarov osteosynthesis for congenital pseudarthrosis of the tibia: an interim report. J Korean Orthop Assoc 2006;41:891-8.

4. Kristiansen LP, Steen H, Terjesen T. Residual challenges after healing of congenital pseudarthrosis in the tibia. Clin Orthop 2003;414:228-37.

5. Gilbert A, Brockman R. Congenital pseudarthrosis of the tibia: long-term follow-up of 29 cases treated by microvascular bone transfer. Clin Orthop 1995;314:37-44.
6. Guidera KJ, Raney EM, Ganey T, et al. Ilizarov treatment of congenital pseudarthrosis of the tibia. J Pediatr Orthop 1997;17:668-74.

7. Ghanem I, Damsin JP, Carlioz H. Ilizarov technique in the treatment of congenital pseudarthrosis of the tibia. J Pediatr Orthop 1997;17:685-90.

8. Boero S, Catagni M, Donzelli O, Facchini R, Frediani V. Congenital pseudarthrosis of the tibia associated with neurofibromatosis. 1: treatment with llizarov's device. J Pediatr Orthop 1997;17:675-84.

9. Kim HW, Weinstein SL. Intramedullary fixation and bone grafting for congenital pseudarthrosis of the tibia. Clin Orthop 2002;405:250-7.

10. Joseph B, Somaraju VV, Shetty SK. Management of congenital pseudarthrosis of the tibia in children under 3 years of age: effect of early surgery on union of the pseudarthrosis and growth of the limb. J Pediatr Orthop 2003;23:740-6.

11. Johnston CE Jr. Congenital pseudarthrosis of the tibia: results of technical variations in the Charnley-Williams procedure. J Bone Joint Surg [Am] 2002;84-A:1799810.

12. Dobbs MB, Rich MM, Gordon JE, Szymanski DA, Schoenecker PL. Use of an intramedullary rod for treatment of congenital pseudarthrosis of the tibia: a long-term follow-up study. J Bone Joint Surg [Am] 2004:86-A:1186-97.

13. Boyd HB. Pathology and natural history of congenital pseudarthrosis of the tibia. Clin Orthop 1982;166:5-13.

14. Crawford AH, Schorry EK. Neurofibromatosis update. J Pediatr Orthop 2006;26:413-23.

15. Baker JK, Cain TE, Tullos HS. Intramedullary fixation for congenital pseudarthrosis of the tibia. J Bone Joint Surg [Am] 1992;74-A:169-78.

16. Coleman SS, Coleman DA. Congenital pseudarthrosis of the tibia: treatment by transfer of the ipsilateral fibula with vascular pedicle. J Pediatr Orthop 1994;14:15660

17. Dormans JP, Krajbich JI, Zuker R, Demuynk M. Congenital pseudarthrosis of the tibia: treatment with free vascularized fibular grafts. J Pediatr Orthop 1990;10:623-8.

18. Weiland AJ, Weiss AP, Moore JR, Tolo VT. Vascularized fibular grafts in the treatment of congenital pseudarthrosis of the tibia. J Bone Joint Surg [Am]1990;72-A:65462

19. Fabry G, Lammens J, Van Melkebeek J, Stuyck J. Treatment of congenital pseudarthrosis with the llizarov technique. J Pediatr Orthop 1988;8:67-70.

20. Plawecki S, Carpentier E, Lascombes P, Prevot J, Robb JE. Treatment of congenital pseudarthrosis of the tibia by the llizarov method. J Pediatr Orthop 1990;10:786-90.

21. Tudisco C, Bollini G, DungI P, et al. Functional results at the end of skeletal growth in 30 patients affected by congenital pseudarthrosis of the tibia. J Pediatr Orthop $B$ 2000;9:94-102.

22. Boyd HB, Sage FP. Congenital pseudarthrosis of the tibia. J Bone Joint Surg [Am] 1958:40-A:1245-70.

23. Grill F, Bollini G, Dungl P, et al. Treatment approaches for congenital pseudarthrosis of tibia: Results of the EPOS multicenter study. J Pediatr Orthop B 2000;9:75-89

24. Ippolito E, Corsi A, Grill F, Wientroub S, Bianco P. Pathology of bone lesions associated with congenital pseudarthrosis of the leg. J Pediatr Orthop B2000:9:3-10. 